\title{
LES of turbulent combustion: On the consistency between flame and flow filter scales
}

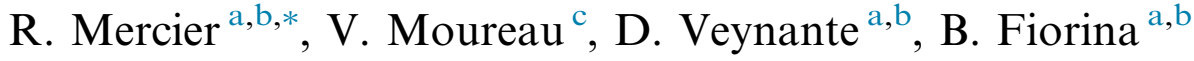 \\ ${ }^{a}$ Ecole Centrale Paris, Grande Voie des Vignes, 92290 Châtenay-Malabry, France \\ ${ }^{\mathrm{b}}$ CNRS, UPR 288, Laboratoire d'Energétique Moléculaire et Macroscopique, Combustion (EM2C), Grande Voie \\ des Vignes, 92290 Châtenay-Malabry, France \\ ${ }^{\mathrm{c}}$ CORIA, UMR 6614, Normandie Université-CNRS, Université et INSA de Rouen, Technopole du Madrillet, \\ BP 8, 76801 Saint-Etienne-du-Rouvray, France
}

\begin{abstract}
A recurrent issue in premixed combustion LES is that the flame thickness is smaller than the grid size. Broadening of the reactive layer is then mandatory to ensure a proper propagation of the filtered flame front. The reactive flow governing equations exhibit then two filter operators of different sizes dedicated to the flow field and the flame front, respectively. The consistency issues between flame and flow filter sizes in LES of turbulent premixed flames are discussed in the present article. A general mathematical formalism considering two different filter sizes is proposed. A new closure of the resulting LES balance equations system is derived in the framework of the Filtered TAbulated Chemistry for LES (F-TACLES) formalism. The new model, called F2-TACLES, is first validated by computing 1-D premixed filtered flames. Then an LES of the complex turbulent premixed PRECCINSTA swirl burner is successfully performed. In particular, the new model formulation improves the prediction of resolved flame/turbulence interactions.
\end{abstract}

\section{Introduction}

An efficient modeling approach for Large Eddy Simulation (LES) of turbulent premixed combustion is to identify the flame front as a propagating surface. The G-equation formalism tracks this surface assumed infinitely thin using level-set numerical techniques [1-4]. Despite a simplified description of the chemical processes, this approach is commonly used in LES of premixed combustion [1,3-5].

Infinitely thin surface approaches however remain limited as they do not describe the inner flame structure. Accounting for the flame thickness is necessary to access thermochemical variables within the flame front but raises several issues in LES of premixed combustion. Figure 1 illustrates the propagation of a filtered flame front in a turbulent environment, using a mesh grid of size $\Delta_{x}$. According to [6], the velocity field $\widetilde{\mathbf{u}}$, Favre-filtered by a Gaussian function of width 


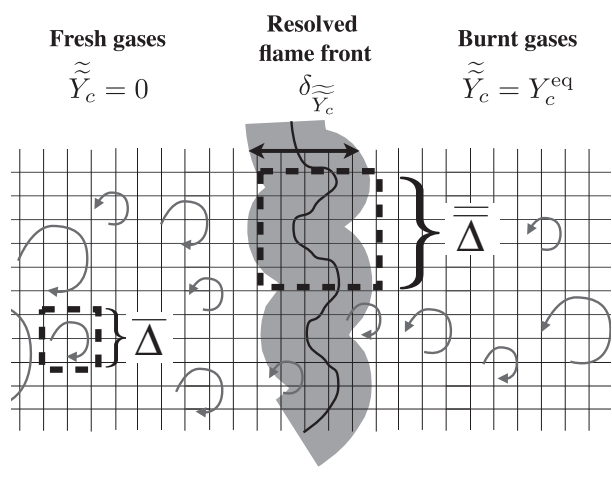

Fig. 1. Filter size issues in turbulent combustion LES.

$\bar{\Delta}$, is well resolved on the LES grid when the resolution criteria $\bar{\Delta}>2 \Delta_{x}$ is satisfied. The flame is here modeled by a progress variable $Y_{c}$, monotonically increasing from 0 in the fresh gases to $Y_{c}^{e q}$ in the burnt gases. In practical meshes, the flame thickness, defined as $\delta_{Y_{c}}=Y_{c}^{\mathrm{eq}} / \max \left(\left|\nabla Y_{c}\right|\right)$, is thinner than the mesh size $\Delta_{x}[7,8]$. Therefore, filtering the progress variable field at the width $\bar{\Delta}$ introduces a resolved flame front of thickness $\delta_{\widetilde{Y}_{c}} \approx \bar{\Delta}$ with $\bar{\Delta} \approx 3 \Delta_{x}$. As further illustrated in Section 2.1, the chemical reaction layer is then under-resolved inducing a misprediction of the flame propagation speed $[9,10]$.

Two major alternatives are then possible to capture both the flame propagation and the flame structure. The first is to artificially thicken the flame front (Thickened Flame model for LES) and to model sub-grid scale (SGS) wrinkling [11]. Efficient and robust, the TFLES model can be combined either with simple [12] or tabulated chemistry $[10,13]$ formalisms. An alternative is to introduce a filter of size $\overline{\bar{\Delta}}>\bar{\Delta}$ dedicated to the flame front. Initially developed for infinitely thin flame front and for single-step chemistry [14-16], this strategy has recently been extended to tabulated chemistry by the Filtered Tabulated Chemistry for LES (F-TACLES) model $[9,17]$. Simulations of propagating filtered premixed planar flames show that the flame resolution criterion $\overline{\bar{\Delta}} / \Delta_{x}$ depends on numerical conditions [18]. For instance, the criteria $\overline{\bar{\Delta}} / \Delta_{x} \geqslant 8$ is required in [9] to predict the proper propagation speed of a laminar premixed filtered flame.

Filtering both flow and thermochemical variables at a unique scale $\overline{\bar{\Delta}}$ would not take full benefit of the grid resolution out of the reaction layer. Therefore, turbulent premixed combustion LES involves, implicitly or explicitly, two filter operators of size $\overline{\bar{\Delta}}$ and $\bar{\Delta}$. The condition $\overline{\bar{\Delta}}>\bar{\Delta}$ is fulfilled in practical combustion LES. The influ- ence of two different filter operators on a combustion LES mathematical formalism has never been studied to our knowledge. The mathematical formalism of the general problem is first developed. Two different strategies to treat differences between flame and flow filters are then proposed in the framework of the F-TACLES model. Both model formulations are then tested on the LES of a swirling premixed flame for which experiments [19] and Direct Numerical Simulations [20] are available.

\section{Two-scales filtering in turbulent combustion LES}

\subsection{Problem formalism}

$\bar{\Phi}$ and $\widetilde{\Phi}$ denote the Reynolds and Favre filtering of a variable $\Phi$ at size $\bar{\Delta}$ while $\overline{\bar{\Phi}}$ and $\widetilde{\widetilde{\Phi}}$ are defined as the values of $\Phi$ filtered at size $\overline{\bar{\Delta}}$. Filtering continuity and momentum equations at the filter scale $\bar{\Delta}$ leads to the following system:

$$
\begin{aligned}
& \frac{\partial \bar{\rho}}{\partial t}+\nabla \cdot(\bar{\rho} \tilde{\mathbf{u}})=0 \\
& \frac{\partial \bar{\rho} \tilde{\mathbf{u}}}{\partial t}+\nabla \cdot(\bar{\rho} \tilde{\mathbf{u}} \tilde{\mathbf{u}})=-\nabla \bar{P}+\nabla \cdot\left(\bar{\tau}+\bar{\tau}^{t}\right)
\end{aligned}
$$

where $\bar{\rho}$ and $\tilde{\mathbf{u}}$ are the filtered density and velocity vector, respectively. $\bar{\tau}$ is the filtered viscous tensor and $\bar{\tau}^{t}=-\bar{\rho}(\widetilde{\mathbf{u u}}-\tilde{\mathbf{u}} \tilde{\mathbf{u}})$ the Reynolds stresses. In a low-Mach number context, $P$ denotes the hydrodynamic pressure.

Considering reactive flows, the propagation of a flame front is governed by the chemical species balance equation:

$\frac{\partial \rho Y_{k}}{\partial t}+\nabla \cdot\left(\rho \mathbf{u} Y_{k}\right)=-\nabla \cdot\left(\rho Y_{k} V_{k}\right)+\rho \dot{\omega}_{Y_{k}}$

where $Y_{k}, \dot{\omega}_{Y_{k}}$ and $V_{k}$ are the mass fraction, the chemical reaction rate and the molecular diffusion velocity of the $k$-th species, respectively. Assuming steady state in the flame coordinate system, the integration of Eq. 3 in the direction $x_{n}$ normal to the flame front gives the flame consumption speed $S_{l}$ :

$S_{l}=\frac{1}{\rho^{u}\left(Y_{k}^{b}-Y_{k}^{u}\right)} \int_{-\infty}^{+\infty} \rho \dot{\omega}_{Y_{k}} d x_{n}$

where superscripts $u$ and $b$ refer to fresh and burnt gases states, respectively. Equation (4) shows that a reliable computation of the flame surface propagation requires a fine numerical resolution of the chemical reaction rate $\dot{\omega}_{Y_{k}}$ involved in the integral of the RHS. This issue is illustrated in Fig. 2 showing the $\mathrm{CO}_{2}$ chemical reaction rate $\rho \dot{\omega}_{\mathrm{Y}_{\mathrm{CO}}}$ normalized by its maximum value and extracted from a 1-D laminar premixed $\mathrm{CH}_{4}$-air flame computed using a detailed chemical scheme [21]. This 
numerical flame solution, resolved on a very fine grid, serves as a reference. Squares symbols, separated by a constant distance $\Delta_{x}=0.5 \mathrm{~mm}$, are superimposed to the chemical reaction rate profile. It illustrates the position of a virtual grid of size $\Delta_{x} \approx \delta_{Y_{c}}$ representative of practical combustion LES. The chemical reaction rate is then filtered at two different sizes $\left(\bar{\Delta}=3 \Delta_{x}\right.$ and $\left.\overline{\bar{\Delta}}=10 \Delta_{x}\right)$ and normalized by the maximum filtered values. In Fig. 2, the reference filtered chemical reaction rate profiles are shown by the solid lines whereas chemical reaction rate profiles, which would be resolved on the LES grid (assuming first order reconstruction), are shown by dashed lines. The chemical reaction rate $\overline{\rho \dot{\omega}_{\mathrm{Y}_{\mathrm{CO}}}}$, filtered at the size $\bar{\Delta}$, is under-resolved on the LES grid, which would lead to numerical errors in the RHS integral estimation of Eq. (4). However, filtering the flame front at a size $\overline{\bar{\Delta}}>\bar{\Delta}$ correctly describes the profile of $\overline{\overline{\rho \dot{\omega}_{\mathrm{CO}_{2}}}}$. Therefore, a sufficient resolution of the flame front is ensured by filtering chemical species balance equation (3) at a size $\overline{\bar{\Delta}}$ :

$$
\begin{aligned}
\frac{\partial \overline{\bar{\rho}} \widetilde{Y_{k}}}{\partial t}+ & \nabla \cdot\left(\overline{\bar{\rho}} \tilde{\tilde{\mathbf{u}}} \widetilde{\widetilde{Y_{k}}}\right)=-\nabla \cdot\left(\overline{\overline{\rho Y_{k} V_{k}}}\right) \\
& -\nabla \cdot\left(\overline{\bar{\rho}} \widetilde{\widetilde{\overline{\mathbf{u} Y}}}-\overline{\bar{\rho}} \tilde{\tilde{\mathbf{u}} \widetilde{Y}_{k}}\right)+\overline{\overline{\rho \dot{\omega}_{Y_{k}}}}
\end{aligned}
$$

LES of turbulent combustion therefore implies to solve the system of Eqs. (1), (2) and (5), involving simultaneously the two filter scales $\bar{\Delta}$ and $\overline{\bar{\Delta}}$. Two strategies to treat different flow and flame filter scales are presented below.

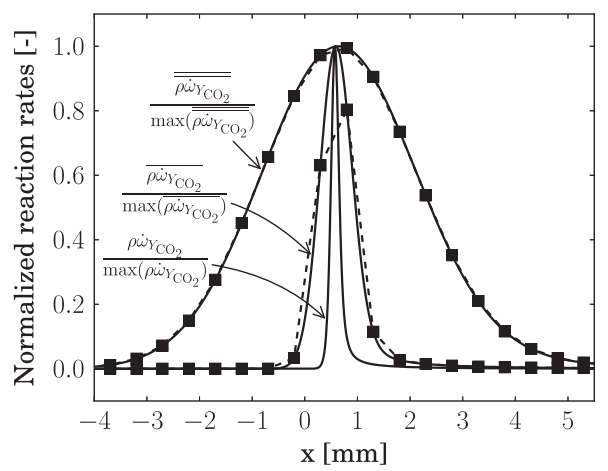

Fig. 2. Comparison of unfiltered and filtered $\mathrm{CO}_{2}$ normalized reaction rates on a virtual LES mesh (squares) of size $\Delta_{x}=0.5 \mathrm{~mm}$. Filter sizes are $\bar{\Delta}=3 \Delta_{x}=1.5 \mathrm{~mm}$ and $\overline{\bar{\Delta}}=10 \Delta_{x}=5 \mathrm{~mm}$. Dashed lines: reconstruction of the reaction rates on the virtual mesh. Solid lines: exact reaction rates.

\subsection{Classical approach: ad hoc coupling of flame and flow filtered equations}

The first strategy is to implicitly substitute the filtered velocity fields $\tilde{\mathbf{u}}$ and $\tilde{\tilde{\mathbf{u}}}$ by a unique variable

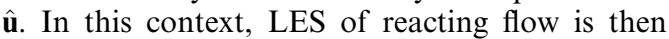
governed by the following set of equations:

$$
\begin{aligned}
& \frac{\partial \overline{\bar{\rho}}}{\partial t}+\nabla \cdot(\overline{\bar{\rho}} \hat{\mathbf{u}})=0 \\
& \frac{\partial \overline{\bar{\rho}} \hat{\mathbf{u}}}{\partial t}+\nabla \cdot(\overline{\bar{\rho}} \hat{\mathbf{u}} \hat{\mathbf{u}})=-\nabla \bar{P}+\nabla \cdot\left(\bar{\tau}+\bar{\tau}^{t}\right) \\
& \frac{\partial \overline{\bar{\rho}} \widetilde{\widetilde{Y_{k}}}}{\partial t}+\nabla \cdot\left(\overline{\bar{\rho}} \hat{\mathbf{u}} \widetilde{\widetilde{Y_{k}}}\right)=-\nabla \cdot\left(\overline{\overline{\rho Y_{k} V_{k}}}\right) \\
& \quad-\nabla \cdot\left(\overline{\bar{\rho}} \widetilde{\widetilde{\mathbf{u} Y_{k}}}-\overline{\bar{\rho}} \hat{\mathbf{u}} \widetilde{\widetilde{Y_{k}}}\right)+\overline{\overline{\rho \dot{\omega}_{Y_{k}}}} \\
& P_{0}=\overline{\bar{\rho}} \widetilde{\widetilde{r T}}
\end{aligned}
$$

where the thermodynamic pressure $P_{0}$ is assumed constant in space for low-Mach number flows. $r=R / W$ where $R$ is the ideal gas constant and $W$ the mean molecular weight. The system of Eqs. (6)-(9), widely used in the literature, remains however ad hoc because it cannot be mathematically derived from the Navier-Stokes equations. The density $\overline{\bar{\rho}}$, the term $\widetilde{\widetilde{r T}}$ and the chemical species $\widetilde{Y_{k}}$, are filtered at the scale $\overline{\bar{\Delta}}$ for an adequate numerical resolution of the flame front. However, hydrodynamic pressure $\bar{P}$, viscous tensor $\bar{\tau}$ and Reynolds stresses $\bar{\tau}^{t}$ are filtered at $\bar{\Delta}$ which is sufficient for resolved flow field variables. The variable $\hat{\mathbf{u}}$ satisfies the two following asymptotic behaviors.

First, far from the reaction zone, the filtered velocity field $\hat{\mathbf{u}}$, solution of Eq. (7), is not influenced by the flame. In the context of premixed combustion, as $\overline{\bar{\rho}}$ is constant in fresh and burnt gases, the filter size associated to the velocity field is $\bar{\Delta}$ and $\hat{\mathbf{u}}=\tilde{\mathbf{u}}$.

Second, across the reactive layer, flame thermochemical variables and density are filtered at the width $\overline{\bar{\Delta}}$. The heat expansion retroacts on the velocity $\hat{\mathbf{u}}$. When the flame structure is laminar, the filter size associated to the velocity $\hat{\mathbf{u}}$ is then $\overline{\bar{\Delta}}$ and $\hat{\mathbf{u}}=\tilde{\tilde{\mathbf{u}}}$. The conservation equation system (Eqs. (6)-(9)) written in the flame referential also correctly degenerates towards laminar flame regime.

However, considering the general case of a turbulent premixed flame, the filter size implicitly associated to $\hat{\mathbf{u}}$ is not clearly identified. Indeed, the impact of the flow patterns resolved at the scale $\bar{\Delta}$ on the flame front filtered at $\overline{\bar{\Delta}}$ may bias the description of flame/turbulence interactions $[2,22]$. The velocity $\hat{\mathbf{u}}$ within the flame front is affected by two different phenomena: (i) the turbulent SGS fluxes, which are modeled by an implicit 
filter of size $\bar{\Delta}[6,9,15,16]$; (ii) thermal expansion, induced by the density filtered at $\overline{\bar{\Delta}}$. This modeling issue is intrinsic to most turbulent combustion models designed to capture the filtered flame thickness. For instance, it is the case for both thickening [11] and filtering [23] approaches developed to properly resolve the flame front. A dedicated approach is mandatory to ensure the consistency between SGS turbulence model and turbulent combustion model within the flame front.

\subsection{New approach: consistent coupling of flame and flow filtered equations}

An alternative is to transport $\widetilde{\widetilde{Y}_{k}}$ by the turbulent flow field filtered at $\bar{\Delta}$. First, the transport equation of $\widetilde{\widetilde{Y_{k}}}$ by $\bar{\rho} \tilde{\mathbf{u}}$ can be derived in a non-conservative form using the total mass conservation at scale $\bar{\Delta}$ (Eq. 1):

$\frac{\partial \bar{\rho} \widetilde{\widetilde{Y}_{k}}}{\partial t}+\nabla \cdot\left(\bar{\rho} \tilde{\mathbf{u}} \widetilde{\widetilde{Y_{k}}}\right)=\bar{\rho} \frac{\partial \widetilde{\widetilde{Y_{k}}}}{\partial t}+\bar{\rho} \tilde{\mathbf{u}} \cdot \nabla \widetilde{\widetilde{Y_{k}}}$

$\bar{\rho} \partial \widetilde{\widetilde{Y}_{k}} / \partial t$ is deducted from the non-conservative form of Eq. (5):

$$
\begin{aligned}
\frac{\partial \bar{\rho} \widetilde{\widetilde{Y_{k}}}}{\partial t}+ & \nabla \cdot\left(\bar{\rho} \tilde{\mathbf{u}} \widetilde{\widetilde{Y_{k}}}\right)=-\frac{\overline{\bar{\rho}}}{\overline{\bar{\rho}}} \nabla \cdot\left(\overline{\overline{\rho Y_{k} V_{k}}}\right) \\
& -\overline{\bar{\rho}} \nabla \cdot\left(\overline{\bar{\rho}} \widetilde{\widetilde{\mathbf{u}_{k}}}-\overline{\bar{\rho}} \widetilde{\widetilde{\mathbf{u}}} \widetilde{\widetilde{Y_{k}}}\right) \\
& -\bar{\rho}(\tilde{\tilde{\mathbf{u}}}-\tilde{\mathbf{u}}) \cdot \nabla \widetilde{\widetilde{Y_{k}}}+\overline{\widetilde{\rho}}
\end{aligned}
$$

Equation (11) RHS exhibits four terms. $\nabla \cdot\left(\overline{\overline{\rho Y_{k} V_{k}}}\right)$ and $\nabla \cdot\left(\overline{\bar{\rho}} \widetilde{\widetilde{\mathbf{u} Y_{k}}}-\overline{\bar{\rho}} \tilde{\tilde{\mathbf{u}}} \widetilde{\widetilde{Y_{k}}}\right)$ are the laminar diffusion and convective fluxes occurring at subscale $\overline{\bar{\Delta}}$, both being weighted by the ratio $\bar{\rho} / \overline{\bar{\rho}} . \bar{\rho} \widetilde{\widetilde{\dot{\omega}_{Y_{k}}}}$ is the chemical reaction rate associated to the $k$-th species filtered at size $\overline{\bar{\Delta}}$. Finally, $\left(\bar{\rho}(\tilde{\tilde{\mathbf{u}}}-\tilde{\mathbf{u}}) \cdot \nabla \widetilde{\widetilde{Y}_{k}}\right)$ is the inter-scale convection term associated to the scales between $\overline{\bar{\Delta}}$ and $\bar{\Delta}$. This term, which vanishes when flame and flow fields are filtered at the same size $\overline{\bar{\Delta}}$, can be either computed directly from the explicit filtering of resolved quantities or modeled as suggested in Section 3.

The analytical integration of Eq. (11) can be performed in the flame coordinate system and across the filtered flame font, assuming that no flame wrinkling occurs. Some mathematical manipulations show an important LES combustion model property, which is that the solution of Eq. (11) is then a planar filtered flame front which propagates at the laminar consumption speed $S_{l}$.

The reactive flow is then described by the chemical species balance equation (Eq. (11)), completed with the flow governing equations given by Eqs. (1) and (2) and with the ideal gas law filtered at size $\bar{\Delta}$. Both the density $\bar{\rho}$ and the temperature $\widetilde{T}$ are therefore filtered at a size $\bar{\Delta}$ $\underline{\underline{\Delta}}$ and the propagation of the flame front filtered at $\bar{\Delta}$ is ensured by Eq. (11). A modeling strategy to handle the closure of resulting equation system at both $\bar{\Delta}$ and $\bar{\Delta}$ filter sizes is now proposed.

\section{Closure of the two-scale filtered progress variable balance equation: F2-TACLES model}

A closure of the formalism detailed in Section 2.3 is now proposed using the F-TACLES formalism [9] mapping the filtered flame structure using 1-D filtered laminar premixed flames. The progress variable $\widetilde{\widetilde{Y}_{c}}$ is defined from filtered species mass fractions $\widetilde{\widetilde{Y}_{k}}$ as: $\widetilde{Y_{c}}=\sum_{k=1}^{N_{s p}} n_{k} \widetilde{\widetilde{Y}_{k}}$ where $n_{k}$ is the coefficient associated to the $k$-th species and $N_{s p}$ the number of species in the chemical scheme. Applying the F-TACLES strategy [9] leads to the following equations:

$$
\begin{aligned}
& \frac{\partial \bar{\rho}}{\partial t}+\nabla \cdot(\bar{\rho} \tilde{\mathbf{u}})=0 \\
& \frac{\partial \bar{\rho} \tilde{\mathbf{u}}}{\partial t}+\nabla \cdot(\bar{\rho} \tilde{\mathbf{u}} \tilde{\mathbf{u}})=-\nabla \bar{P}+\nabla \cdot\left(\bar{\tau}+\bar{\tau}^{t}\right) \\
& \frac{\partial \bar{\rho} \widetilde{Y_{c}}}{\partial t}+\nabla \cdot\left(\bar{\rho} \tilde{\mathbf{u}} \widetilde{\widetilde{Y}}_{c}\right)=\Xi_{\bar{\Delta}} \nabla \cdot\left(\rho_{0} D_{0} \nabla \widetilde{\widetilde{Y}_{c}}\right) \\
& \quad+\Xi_{\bar{\Delta}} \Sigma_{Y_{c}}^{\mathrm{F} 2 \mathrm{~T}}\left[\widetilde{\widetilde{Y}_{c}}\right] \\
& P_{0}=\widetilde{\rho} r T^{*}\left[\widetilde{\widetilde{Y}_{c}}\right]
\end{aligned}
$$

where the notation $\Phi[A]$ denotes that $\Phi$ is tabulated as a function of $A . \rho_{0}$ and $D_{0}$ are reference values for the density and the molecular diffusion coefficient. $\Xi_{\overline{\bar{\Delta}}}$ is the subgrid scale wrinkling model associated to the resolved filtered flame front at scale $\overline{\bar{\Delta}}$. In the present study, $\Xi_{\overline{\bar{\Delta}}}$ is modeled using the analytical formulation proposed in $[25,26]$ but may also be closed using more recent dynamic approaches [27]. The term $\quad \Sigma_{Y_{c}}^{\mathrm{F} 2 \mathrm{~T}}\left[\widetilde{\widetilde{Y}}_{c}\right]=\mathcal{D}_{Y_{c}}$ $+\mathcal{T}_{Y_{c}}+\mathcal{I}_{Y_{c}}+\mathcal{R}_{Y_{c}}$ is tabulated as a function of the resolved filtered progress variable $\widetilde{\widetilde{Y}}_{c}$ for a given pair $(\overline{\bar{\Delta}}, \bar{\Delta})$. The laminar diffusion correction term $\mathcal{D}_{Y_{c}}$, which accounts for differential diffusion in the direction normal to the flame front, reads: 


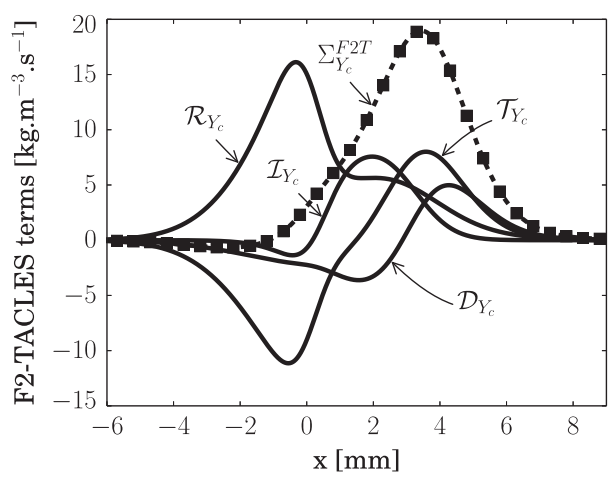

Fig. 3. F2-TACLES model terms, computed from a $\mathrm{CH}_{4}$-air 1-D premixed flame using detailed chemistry [21], for $\bar{\Delta}=1.75 \mathrm{~mm}$ and $\overline{\bar{\Delta}}=7 \mathrm{~mm}$. A virtual LES mesh (squares) of size $\Delta_{x}=0.5 \mathrm{~mm}$ is added to the global source term $\sum_{Y_{c}}^{F 2 T}$ profile.

$\mathcal{D}_{Y_{c}}=-\left(\overline{\overline{\bar{\rho}^{*}}} \frac{\partial}{\partial x^{*}}\left(\overline{\overline{\sum_{k=1}^{\rho_{s p}} n_{k} \rho^{*} Y_{k}^{*} V_{k}^{*}}}\right)+\frac{\partial}{\partial x^{*}}\left(\rho_{0} D_{0} \frac{\partial \widetilde{\widetilde{Y_{c}^{*}}}}{\partial x^{*}}\right)\right)$

where the superscript * stands for variables extracted from a 1-D laminar premixed flame. The laminar SGS convective fluxes, modeled by $\mathcal{T}_{Y_{c}}$, are also estimated from the chemical structure of a 1-D filtered laminar premixed flame:

$\mathcal{T}_{Y_{c}}=-\rho_{0} S_{l}^{0} \frac{\rho^{*}}{\overline{\overline{\rho^{*}}}}\left(\frac{\partial \overline{\overline{Y_{c}^{*}}}}{\partial x^{*}}-\frac{\partial \widetilde{\widetilde{Y_{c}^{*}}}}{\partial x^{*}}\right)$

This strategy is also applied to model the interscale convection term:

$\mathcal{I}_{Y_{c}}=\rho_{0} S_{l}^{0}\left(1-\frac{\overline{\rho^{*}}}{\overline{\overline{\rho^{*}}}}\right) \frac{\partial \widetilde{\widetilde{Y_{c}^{*}}}}{\partial x^{*}}$

Finally, the filtered progress variable reaction rate is estimated as $\mathcal{R}_{Y_{c}}=\widetilde{\rho^{*}} \widetilde{\widetilde{\dot{\omega}_{Y_{c}}^{*}}}$. In practice, the term $\Sigma_{Y_{c}}^{\mathrm{F} 2 \mathrm{~T}}$ is pre-computed and stored in a look-up table as a function of $\underset{\widetilde{Y}_{c}}{\widetilde{T}}$. The present modeling strategy, which combines two filter length scales $\bar{\Delta}$ and $\overline{\bar{\Delta}}$ explicitly in the same filtered look-up table, is called F2-TACLES. As an illustration, these terms have been computed across a 1-D premixed laminar $\mathrm{CH}_{4}$-air flame of equivalence ratio 0.83. They are plotted in Fig. 3 as a function of space coordinate $x$ for $\bar{\Delta}=1.75 \mathrm{~mm}$ and $\overline{\bar{\Delta}}=7 \mathrm{~mm}$. In particular, it is shown that $\Sigma_{Y_{c}}^{\mathrm{F} 2 \mathrm{~T}}$ is well resolved on a grid of size $\Delta_{x}=0.5 \mathrm{~mm} \approx \delta_{Y_{c}}=0.46 \mathrm{~mm}$. Other thermochemical variables, such as chemical species mass fractions $Y_{k}$ can also be filtered at $\bar{\Delta}$ and tabulated as a function of the resolved progress variable $\widetilde{\widetilde{Y}_{c}}$ as $\widetilde{Y_{k}}=\widetilde{Y_{k}^{*}}\left[\widetilde{\widetilde{Y}_{c}}\right]$. The choice of the pair $(\overline{\bar{\Delta}}, \bar{\Delta})$ used to generate the filtered chemical database is now discussed in Section 4.1.

\section{Numerical applications}

\subsection{1-D filtered flame simulations}

F2-TACLES terms are computed explicitly prior to the LES, then both flow $\bar{\Delta}$ and flame $\overline{\bar{\Delta}}$ filter sizes should be prescribed at the database generation and chosen carefully. First, $\bar{\Delta} \approx 3 \Delta_{x}$ is the filter size associated to the thermochemical variables $\widetilde{T}$ and $\bar{\rho}$. A too small value of $\bar{\Delta}$ would cause numerical problems if the gradient of $\bar{\rho}$ becomes too important. A criterion for $\bar{\Delta} / \Delta_{x}$ depends on the numerical schemes employed in the LES code. The filter associated to the flame front, $\overline{\bar{\Delta}}$, is set to ensure a sufficient resolution of the filtered progress variable source term $\Sigma_{Y_{c}}^{F 2 T}$. The corresponding criterion for $\overline{\bar{\Delta}} / \Delta_{x}$ also depends on the numerical schemes. To go further, several LES of 1-D premixed flames are performed to assess the model properties and determine both minimum flow $\bar{\Delta}$ and flame $\overline{\bar{\Delta}}$ filter sizes for a given mesh. The YALES2 low-Mach number LES solver [28], retained here, uses a centered fourth-order scheme for spatial discretization while time integration of convective terms is performed with a fourth-order temporal scheme. Chemistry is tabulated from a 0.83 equivalence ratio 1-D laminar premixed $\mathrm{CH}_{4}$-air flame computed with the Lindstedt [21] chemical scheme, involving 29 species and 141 reactions. A parametric study on the ratio $\bar{\Delta} / \Delta_{x}$ is first conducted varying the mesh size $\Delta_{x}$ for a constant filter size $\bar{\Delta}=1.75 \mathrm{~mm}$. The width $\overline{\bar{\Delta}}=7 \mathrm{~mm}$ is also set constant and sufficiently large so that $\overline{\bar{\Phi}}$ quantities are always well resolved $\left(\delta_{\widetilde{\tilde{Y}}_{c}}=4.3 \mathrm{~mm}\right)$. For each computation, the ratio of the effective flame propagation speed $S_{\Delta}$ and the laminar flame speed $S_{l}^{0}$ is plotted as a function of $\bar{\Delta} / \Delta_{x}$ in Fig. 4 and shows
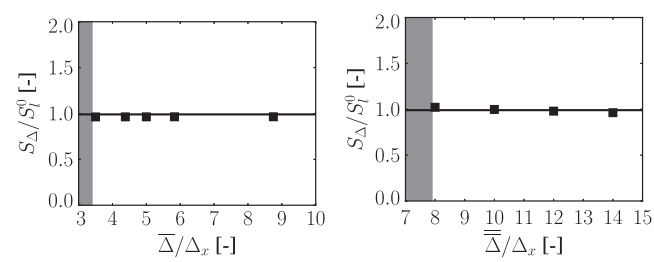

Fig. 4. Left: $S_{\Delta} / S_{l}^{0}$ in terms of $\bar{\Delta} / \Delta_{x}$ with $\bar{\Delta}=1.75 \mathrm{~mm}$ and $\overline{\bar{\Delta}}=7 \mathrm{~mm}$. Right: $S_{\Delta} / S_{l}^{0}$ in terms of $\overline{\bar{\Delta}} / \Delta_{x}$ with $\bar{\Delta}=1.75 \mathrm{~mm}$ and $\Delta_{x}=0.5 \mathrm{~mm}$. Inside the gray surface, simulation where unstable and the predicted flame speed value was meaningless. 
that the limiting criterion associated to the YALES 2 code is $\bar{\Delta} / \Delta_{x} \geqslant 3.5$. Below this limit, the numerical scheme diverges and solutions are meaningless. A second parametric study on the ratio $\overline{\bar{\Delta}} / \Delta_{x}$ varying the flame filter size $\overline{\bar{\Delta}}$ for constant flow filter $\bar{\Delta}=1.75 \mathrm{~mm}$ and mesh $\Delta_{x}=0.5 \mathrm{~mm}$ sizes (respecting the criteria $\bar{\Delta} / \Delta_{x} \geqslant 3.5$ ) shows that the flame filter size should verify $\overline{\bar{\Delta}} / \Delta_{x} \geqslant 8.0$ for the YALES 2 code. This criterion may be recast as $\delta_{\tilde{c}} / \Delta_{x} \geqslant 5.0$ matching the limiting criterion of the original F-TACLES model [9].

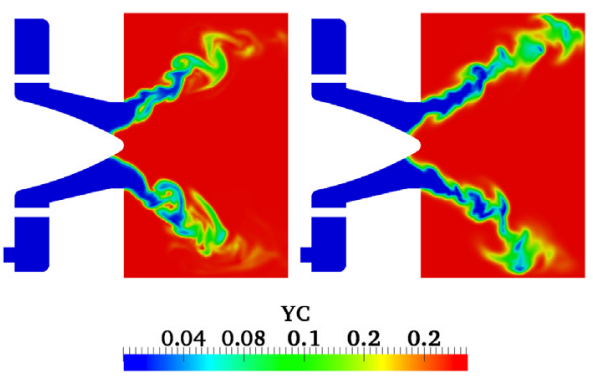

(a) Left: $\widetilde{\widetilde{Y}}_{c}$ (F-TACLES); Right: $\tilde{\widetilde{Y}}_{c}$ (F2TACLES).
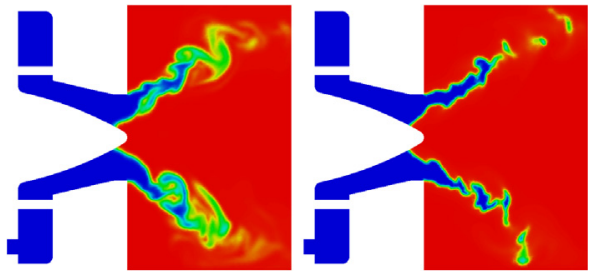

\section{$\begin{array}{lllll}400 & 800 & 1200 & 1600 \quad 2000\end{array}$}

(b) Left: $\widetilde{\widetilde{T}}$ (F-TACLES); Right: $\widetilde{T}$ (F2TACLES)
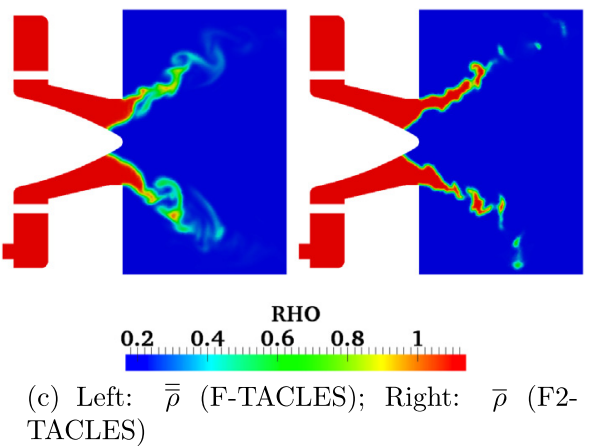

Fig. 5. 2-D color mapping of instantaneous thermochemical variables extracted from LES of the PRECCINSTA burner using F-TACLES (left) and F2-TACLES (right) models.

\subsection{LES of a turbulent swirl combustor}

The F2-TACLES model (Section 3) is now compared to the original F-TACLES model [9] by performing LES of the complex PRECCINSTA swirl burner [19]. Details on the geometry and experimental conditions can be found in $[9,19,20]$. The computational domain comprises a plenum, a swirler, a square combustion chamber and an exhaust pipe. An unstructured tetrahedral mesh (2.5 million nodes) is used for both F-TACLES and F2-TACLES simulations and is representative of an industrial LES grid. The characteristic mesh size in the flame zone is $\Delta_{x} \approx 0.5 \mathrm{~mm}$. For the F2-TACLES model, the flow and flame filter sizes are $\bar{\Delta}=1.75 \mathrm{~mm}$ and $\overline{\bar{\Delta}}=7 \mathrm{~mm}$, respectively, leading to $\delta \widetilde{\widetilde{Y}}_{/} / \Delta_{x}=8.6$. For the original F-TACLES model, the flame filter size is set to $\overline{\bar{\Delta}}=7 \mathrm{~mm}$ as for F2-TACLES. As the burner is fed by a $\phi=0.83$ equivalence ratio $\mathrm{CH}_{4}$-air mixture, both the F-TACLES and F2-TACLES filtered databases have been built from the 1-D flame introduced in Section 4.1. The total injected mass flow rate is $\dot{m}=12.9$ g.s ${ }^{-1}$. The burner is supposed fully adiabatic and mixture composition heterogeneities are neglected. For both models, the progress variable is defined as $Y_{c}=Y_{\mathrm{CO}_{2}}+Y_{\mathrm{CO}}$ in order to uniquely describe the thermochemical flame structure at $\phi=0.83$. Figure 5 shows 2-D views of the instantaneous resolved progress variable and temperature for both F-TACLES and F2-TACLES computations. Filtered progress variable fields (Fig. 5(a)) does not exhibit notable differences as both are solved at the filter size $\overline{\bar{\Delta}}$. However, significant differences can be noticed in Fig. 5(b) as filter sizes associated to both $T$ (and $\rho$ ) differ between computations. In the original F-TACLES model, all the thermochemical values are filtered at $\overline{\bar{\Delta}}$ while only the resolved progress variable $\widetilde{Y_{c}}$ is filtered at this size in the F2-TACLES model. Other thermochemical variables as $\bar{\rho}, \widetilde{T}$ and the chemical species $\widetilde{Y_{k}}$ are only filtered at $\bar{\Delta}$ allowing a finer description of the flame front on a given mesh.

Mean and RMS temperature radial profiles are plotted in Fig. 6(a) and (b), respectively. Very good agreement is found for both modeling approaches. Mean predictions are very close near the burner exit (located at $Z=0 \mathrm{~mm}$ ) while F2TACLES provides a better prediction of the mean flame brush thickness further downstream. Same conclusions can be made when looking at temperature fluctuations profiles. In particular, F2TACLES predicts higher resolved RMS compared to F-TACLES. Indeed, as the thermal thickness of the resolved flame front is thinner, more interactions occur with the resolved turbulent motions. Statistics of $\mathrm{CH}_{4}$ mass fraction are shown in 
Fig. 7(a) and (b). A very good agreement is also noted near the burner exit while the predictions from F2-TACLES model better agrees with experiments both for mean and RMS quantities.

\section{Conclusion}

Consistency issues between flame and flow filter sizes in LES of turbulent premixed flames has been discussed leading to a general mathematical formalism considering two different filter sizes. Filtered progress variable balance equation was recast accordingly. A new closure of the

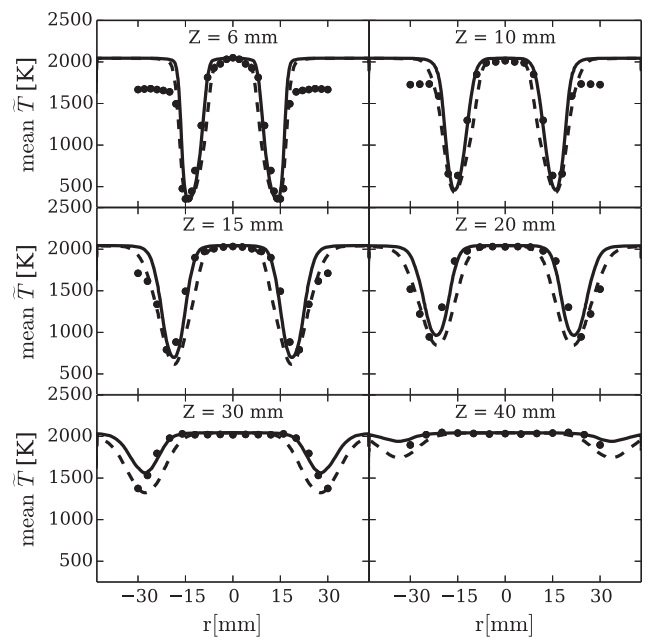

(a) Mean

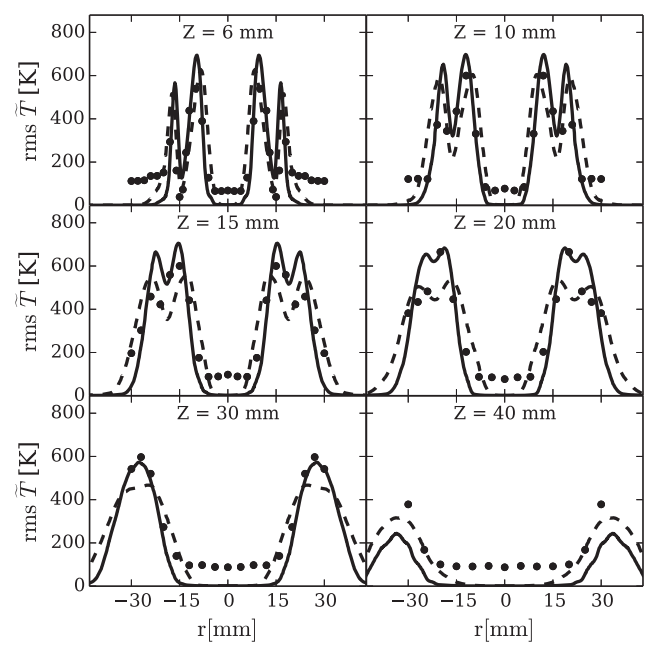

(b) RMS

Fig. 6. Temperature radial profiles from the LES of the PRECCINSTA burner with F-TACLES $(\widetilde{\widetilde{T}}:---)$ and F2-TACLES $(\widetilde{T}$ : - $)$. Experiments: resulting LES balance equation system has been proposed in line with the F-TACLES formalism. This new model, called F2-TACLES, does not induce additional computational cost and its implementation within an LES code remains similar to F-TACLES. Only the tabulation process differs by the computation of the new closure terms. F2-TACLES has been first validated analyzing 1-D premixed filtered flames and through LES of the complex PRECCINSTA swirl burner. Comparisons of mean profiles show that the F2TACLES model better predict the flame brush downstream of the burner exit where the flame-turbulence interactions are more developed. However, mean profiles analysis is probably not

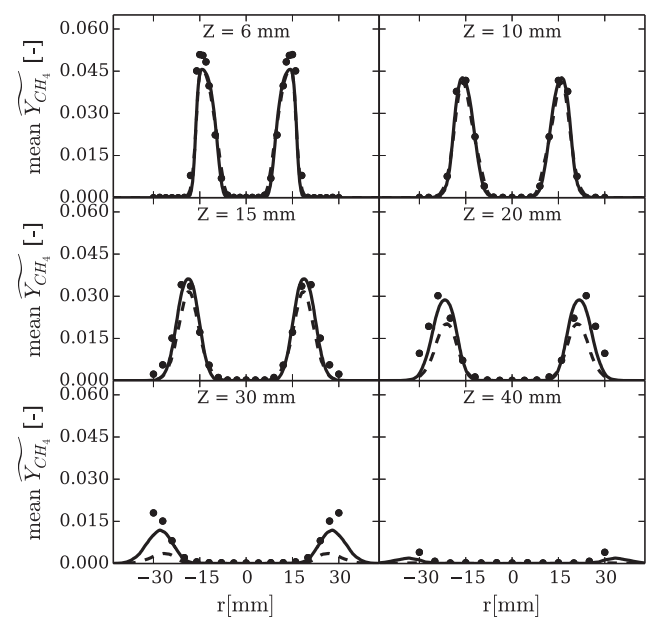

(a) Mean

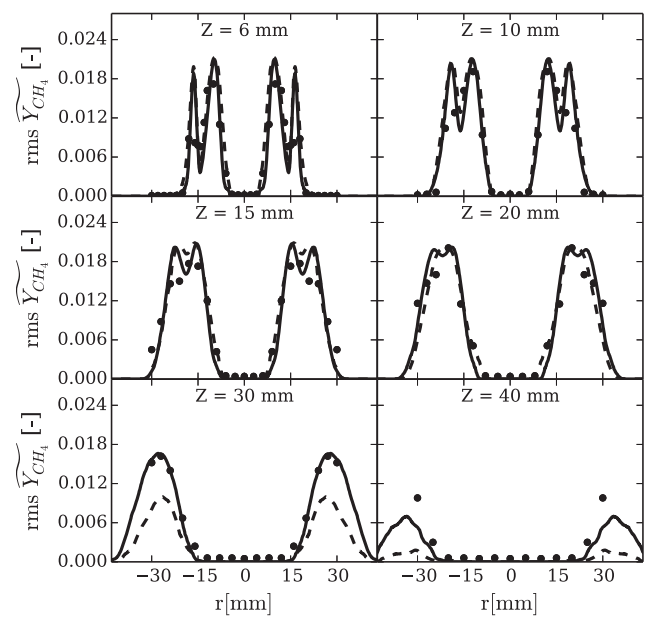

(b) RMS

Fig. 7. $\mathrm{CH}_{4}$ radial profiles from the LES of the PRECCINSTA burner with F-TACLES $\left(\widetilde{\widetilde{Y}}_{\mathrm{CH}_{4}}\right.$ : ---) and F2-TACLES $\left(\widetilde{Y}_{\mathrm{CH}_{4}}:-\right)$. Experiments: $\bullet \bullet$. 
sufficient to fully assess the impact of the new modeling approach. Other configurations, including the characterization of the unsteady behavior of the resolved flows, will be investigated in the future.

\section{References}

[1] S. Menon, W. Jou, Combust. Sci. Tech. 75 (1991) 53-72.

[2] H. Pitsch, Combust. Flame 143 (4) (2005) 587-598ss.

[3] V. Moureau, P. Minot, H. Pitsch, C. Berat, J. Comput. Phys. 221 (2) (2007) 600-614.

[4] V. Moureau, B. Fiorina, H. Pitsch, Combust. Flame 156 (4) (2009) 801-812.

[5] E. Knudsen, H. Pitsch, Combust. Flame 154 (4) (2008) 740-760.

[6] S.B. Pope, Turbulent Flows, Cambridge University Press, 2000.

[7] T. Poinsot, D. Veynante, Theoretical and Numerical Combustion, third ed., 2012, http://elearning.cerfacs.fr/combustion/onlinePoinsotBook/buythirdedition/index.php.

[8] C. Nottin, R. Knikker, M. Boger, D. Veynante, Proc. Combust. Inst. 33 (1), 2011, 1331-1338, http://dx.doi.org/10.1016/j.proci.2010.05.045.

[9] B. Fiorina, R. Vicquelin, P. Auzillon, N. Darabiha, O. Gicquel, D. Veynante, Combust. Flame 157 (3) (2010) 465-475. http://dx.doi.org/10.1016/ j.combustflame.2009.09.015.

[10] G. Kuenne, A. Ketelheun, J. Janicka, Combust. Flame 158 (9) (2011) 1750-1767. http://dx.doi.org/ 10.1016/j.combustflame.2011.01.005.

[11] O. Colin, F. Ducros, D. Veynante, T. Poinsot, Phys. Fluids 12 (7) (2000) 1843-1863.

[12] B. Franzelli, E. Riber, L.Y. Gicquel, T. Poinsot, Combust. Flame 159 (2) (2012) 621-637. http:// dx.doi.org/10.1016/j.combustflame.2011.08.004.

[13] G. Albouze, T. Poinsot, L. Gicquel, C. R. Méc. 337 (6-7) (2009) 319-328.
[14] M. Bouger, D. Veynante, H. Boughanem, A. Trouvé, Proc. Combust. Inst. 27 (1) (1998) 917 925. http://dx.doi.org/10.1016/S0082-0784(98) 80489-X.

[15] C. Duwig, Flow Turbul. Combust. 79 (4) (2007) 433 454.

[16] C. Duwig, Combust. Theory Modell. 13 (2) (2009) 251-268.

[17] P. Auzillon, O. Gicquel, N. Darabiha, D. Veynante, B. Fiorina, Combust. Flame 159 (8) (2012) 2704 2717. http://dx.doi.org/10.1016/j.combustflame. 2012.03.006.

[18] P. Auzillon, B. Fiorina, R. Vicquelin, N. Darabiha, O. Gicquel, D. Veynante, Proc. Combust. Inst. 33 (1) (2011) 1331-1338. http://dx.doi.org/10.1016/ j.proci.2010.05.045.

[19] W. Meier, P. Weigand, X. Duan, R. GiezendannerThoben, Combust. Flame 150 (1-2) (2007) 2-26.

[20] V. Moureau, P. Domingo, L. Vervisch, Combust. Flame 158 (7) (2011) 1340-1357. http://dx.doi.org/ 10.1016/j.combustflame.2010.12.004.

[21] P. Lindstedt, 12 Month Progree Report 1., Tech. Rep. TR-96 009, Brite Euram Program Project BE $951523,1997$.

[22] N. Peters, Turbulent Combustion, Cambridge University Press, 2000.

[23] M. Boger, D. Veynante, in: C. Dopazo (Ed.), Advances in Turbulence, CIMNE, Barcelona, 2000, pp. 449-452.

[24] F. Charlette, C. Meneveau, D. Veynante, Combust. Flame 131 (1/2) (2002) 159-180. http://dx.doi.org/ 10.1016/S0010-2180(02)00401-7.

[25] G. Wang, M. Boileau, D. Veynante, Combust. Flame 158 (11) (2011) 2199-2213. http:// dx.doi.org/10.1016/j.combustflame.2011.04.008.

[26] T. Schmitt, A. Sadiki, B. Fiorina, D. Veynante, Proc. Combust. Inst. 34 (1) (2013) 1261-1268. http://dx.doi.org/10.1016/j.proci.2012.06.150.

[27] V. Moureau, P. Domingo, L. Vervisch, C. R. Méc. 339 (2-3) (2011) 141-148. http://dx.doi.org/ 10.1016/j.crme.2010.12.001. 